\title{
OSCULATING PRIMES TO CURVES OF INTERSECTION IN 4-SPACE, AND TO CERTAIN CURVES IN $n$-SPACE
}

\author{
by R. H. DYE \\ (Received 23rd January 1973)
}

\section{Introduction}

An irreducible curve in $S_{4}$, projective 4-space, may arise as the complete intersection of three given irreducible threefolds. At a simple point $P$ on such a curve there is an osculating solid, and we would like to have its equation. This solid, necessarily containing the tangent line to the curve at $P$, belongs to the net spanned by the tangent solids at $P$ to the threefolds. We seek the appropriate linear combination of the known equations for these tangent solids.

Hesse $\left(9\right.$, p. 283) solved the analogous problem in $S_{3}$; he found the osculating planes to an irreducible curve which is the complete intersection of two surfaces. Salmon (10, p. 328) and Baker (3, p. 206) reproduce Hesse's solution. Later Clebsch (5) found a surface cutting this curve at its contacts with its hyperosculating planes (see also (10), p. 330): for a canonical curve these stalls are the Weierstrass points $(3, \mathrm{pp} .186,201)$.

The problem of finding the equation for the osculating solid in $S_{4}$ was posed recently by Edge (6), who remarks on page 277 that Hesse's methods only carry one a certain distance towards its solution. Indeed, they do not yield a complete solution in the simple case when each threefold is a general quadric. Edge is, however, concerned with a special curve, the octavic intersection of three quadrics with a common self-polar simplex. He finds the osculating solids to this curve by expeditious use of the more amenable algebra (6, p. 278).

The general problem is solved in Section 2 of this paper. Precisely, we obtain the equation for the osculating solid to a complete irreducible curve of intersection of three threefolds in $S_{4}$ at a simple point whose osculating plane has its statutory minimal 3-point contact. Edge's curve has simple points whose osculating planes have 4-point contact $(6$, p. 279$)$. These points come into prominence when we harmonise his and our solutions. Our equation has certain affinities with that of Clebsch's surface. This is because we, like Clebsch, need differentials up to the third order: Hesse required only second order differentials. When each threefold is a quadric their intersection is a canonical curve of genus 5 , so it is a happy coincidence that our equation takes a particularly neat form for this curve. Connected with the equation are three quartic threefolds, and we briefly indicate their geometrical relation to the quadrics. 
Edge asked his question in the classical setting of complex projective geometry, and we shall answer in the same setting. As is usual, the result is valid in an $S_{4}$ over any algebraically closed field of characteristic zero. So we present our solution in a form that can be adapted easily to the more general setting, and we briefly indicate the modifications necessary.

In Section 4 we examine a class of special curves of intersection in $S_{n}$ : the intersection of $(n-1)$ quadrics with a common self-polar simplex is one of these curves. Each curve has special points where the osculating spaces have exceptionally high order of contact; the situation parallels that for Edge's curve. We show, also, that Edge's method extends to yield the equation of the osculating $S_{n-1}$ at a "non-special " point of such a curve.

\section{Osculating solids to curves of intersection in $\mathbf{S}_{\mathbf{4}}$}

2.1. We take $\left(x_{0}, x_{1}, x_{2}, x_{3}, x_{4}\right)=x^{\prime}$ as the coordinates of the $S_{4}$.

Suppose that the irreducible curve $C$ is the complete intersection of the irreducible threefolds: $f(x)=0 ; g(x)=0 ; h(x)=0$; whose respective orders are $p, q, r \geqq 2$. We mean by this that the intersection of the threefolds has a single component, namely $C$, which occurs with multiplicity one; the threefolds intersect simply along $C$. The order of $C$ is pqr, and a special case of Noether's Theorem states that the equations of the threefolds form a basis for the ideal of C.

Let $P$ be a simple point on $C$, and suppose that we know a coordinate vector for $P$. Like Hesse, to aid our calculation we introduce an auxiliary linear form: this is to be any linear form which takes the value 1 when we substitute the vector of $P$ for $x$. Suppose that $\sum_{i} a_{i} x_{i}$ is such a form, where here and throughout Sections 2 and 3 the summations run over $0,1,2,3,4$. If desired, we may choose all but one of the $a_{i}$ to be zero, but we do not wish to specify the nonzero $a_{i}$.

$P$ is simple, so through it passes a single branch of $C$. We take affine (or normalised) coordinates for this branch so that if $\xi$ is the vector of one of its points then

$$
\sum_{i} a_{i} \xi_{i}=1 \text {. }
$$

A minimal or irreducible parametrisation of the branch gives the $\xi_{i}$ as power series in some complex parameter $t$. The sufficiently small $t$ for which the series converge give the points on the branch $(3$, p. $35 ; 11$, pp. 187,$193 ; 13$, pp. 96 , 97).

2.2. The equation for the osculating solid to $C$ at $P$ will involve $5 \times 5$ determinants some of whose entries are themselves $7 \times 7$ determinants. To ease our manipulations it is essential to introduce some notation.

If $v(x)$ is a form then $v_{i}(x)$ denotes its partial derivative with respect to $x_{i}$, and $\nabla v(x)$ is the column 5 -vector whose $(i+1)$ th entry is $v_{i}(x)$ for $i=0,1,2,3,4$. 
We write $F(x)$ for the Hessian matrix $\left(f_{i j}(x)\right)$, with $G(x)$ and $H(x)$ similarly defined.

Using, as previously, dashes to denote transpose we define

$$
\mathscr{F}(x)=(p-1)^{-2} \operatorname{det}\left(\begin{array}{c|c}
F(x) \\
\nabla g(x)^{\prime}
\end{array} \mid \begin{array}{cc}
\nabla g(x), \quad \nabla h(x) \\
\nabla h(x)^{\prime}
\end{array}\right) .
$$

$\mathscr{G}(x)$ and $\mathscr{H}(x)$ are obtained by cyclic change of $f, g, h$. Notice that the form $\mathscr{F}(x)$ has degree $3 p+2 q+2 r-10$.

$F, v, v_{i}, \nabla v$ will denote the results of substituting $\xi$ for $x$ in $F(x), v(x), v_{i}(x)$, $\nabla v(x)$ respectively: $G$ and $H$ have the obvious meanings. In particular we have

and

$$
f=g=h=0,
$$

$$
\begin{array}{r}
\mathscr{F}=(p-1)^{-2} \operatorname{det}\left(\begin{array}{c|c|c|c}
\frac{F}{(\nabla g)^{\prime}} & \nabla g, \quad \nabla h \\
(\nabla h)^{\prime} & 0_{2}
\end{array}\right) ; \mathscr{G}=(q-1)^{-2} \operatorname{det}\left(\begin{array}{c|c}
\frac{G}{(\nabla h)^{\prime}} & \nabla h, \quad \nabla f \\
(\nabla f)^{\prime} & 0_{2}
\end{array}\right) ; \\
\mathscr{H}=(r-1)^{-2} \operatorname{det}\left(\begin{array}{l|ll}
\frac{\nabla}{(\nabla f)^{\prime}} & \nabla f, \quad \nabla g \\
(\nabla g)^{\prime} & 0_{2}
\end{array}\right) .
\end{array}
$$

We write $\left(a_{0}, a_{1}, a_{2}, a_{3}, a_{4}\right)=a^{\prime}$. The determinant of the matrix obtained by removing the $(i+1)$ th row from the $5 \times 4$ matrix $(\nabla f, \nabla g, \nabla h, a)$ will be denoted by $|\nabla f, \nabla g, \nabla h, a|_{i}$.

2.3. In this subsection we collect various formulae necessary to our computation. They hold for $\xi$ on the branch. We differentiate (with respect to $t$ ) (1) and (3) to obtain

$$
a^{\prime} d \xi=(\nabla f)^{\prime} d \xi=(\nabla g)^{\prime} d \xi=(\nabla h)^{\prime} d \xi=0 .
$$

Two further differentiations of (5) give successively

and

$$
\sum_{i} f_{i} d^{2} \xi_{i}+\sum_{i, j} f_{i j} d \xi_{i} d \xi_{j}=0
$$

$$
\sum_{i} f_{i} d^{3} \xi_{i}+\sum_{i, j} f_{i j} d^{2} \xi_{i} d \xi_{j}+d\left(\sum_{i, i} f_{i j} d \xi_{i} d \xi_{j}\right)=0 .
$$

Euler's Theorem for homogeneous functions gives

$$
\begin{gathered}
\xi^{\prime} \nabla f=0, \\
F \xi=(p-1) \nabla f, \\
\xi^{\prime} \nabla \mathscr{F}=(3 p+2 q+2 r-10) \mathscr{F} .
\end{gathered}
$$

When we differentiate (8) and use (5) we find that

$$
\xi^{\prime} d(\nabla f)=0 .
$$

E.M.S. $-18 / 4-F$ 
Finally we have

$$
F d \xi=d(\nabla f) .
$$

There are analogues of (5)-(12) for $g$ and $h$.

2.4. $P$ is simple, so $(\nabla f, \nabla g, \nabla h)$ has rank 3 when $t=0$ (see Section 2.1). From (1) and (8) we infer that $(\nabla f, \nabla g, \nabla h, a)$ has rank 4 at $P$. Hence there is an $i$ for which $|\nabla f, \nabla g, \nabla h, a|_{i}$ is non-zero at $P$. But $|\nabla f, \nabla g, \nabla h, a|_{i}$ is a power series in $t$, so for all sufficiently small $t$ this $|\nabla f, \nabla g, \nabla h, a|_{i}$ is non-zero and its reciprocal is a convergent power series in $t$. From now on we confine our attention to the $\xi$ corresponding to such $t$ : for these $\xi$ the rank of $(\nabla f, \nabla g, \nabla h, a)$ is 4 . We deduce from (5) that

$$
d \xi_{i}=(-1)^{i} \kappa|\nabla f, \nabla g, \nabla h, a|_{i} \text { for } i=0,1,2,3,4,
$$

where $\kappa$ is the product of $d t$ with a convergent power series in $t$, and is independent of $i$. A differentiation gives, when we make an obvious extension of our notation,

$$
\begin{aligned}
& d^{2} \xi_{i}=(-1)^{i} d \kappa \mid \nabla f, \nabla g, \nabla h,\left.a\right|_{i}+(-1)^{i} \kappa|d(\nabla f), \nabla g, \nabla h, a|_{i} \\
&+(-1)^{i} \kappa|\nabla f, d(\nabla g), \nabla h, a|_{i}+(-1)^{i} \kappa|\nabla f, \nabla g, d(\nabla h), a|_{i} .
\end{aligned}
$$

2.5. We can now obtain an expression for $\sum_{i} f_{i} d^{2} \xi_{i}$. A Laplace expansion of the determinant by its last 4 columns and rows taken with (13) shows that

$$
\sum_{i, j} f_{i j} d \xi_{i} d \xi_{j}=\kappa^{2} \operatorname{det}\left(\begin{array}{l|llll}
F & \nabla f, & \nabla g, & \nabla h, \quad a \\
\hline(\nabla f)^{\prime} & & & \\
(\nabla g)^{\prime} & & 0 & \\
(\nabla h)^{\prime} & &
\end{array}\right) .
$$

Subtract from the 6 th column of the determinant $(p-1)^{-1} \xi_{i}$ times the $(i+1)$ th column for $i=0,1,2,3,4$. By (1), (8) and (9) the new 6th column has zero for all its entries apart from the last which is $-(p-1)^{-1}$. Thus the determinant has value

$$
(p-1)^{-1} \operatorname{det}\left(\begin{array}{l|lll}
F & \nabla g, & \nabla h, & a \\
(\nabla f)^{\prime} & \\
(\nabla g)^{\prime} & 0_{3} \\
(\nabla h)^{\prime} &
\end{array}\right) .
$$

Repeating the procedure on the rows we find from (4) and (6) that

$$
\sum_{i} f_{i} d^{2} \xi_{i}=\kappa^{2} \mathscr{F} \text {. }
$$

There are similar formulae for $g$ and $h$. This is true in particular when $t=0$. By Section 2.1 the vector $\xi$ is then the given one for $P$. We obtain 
Lemma 1. If $\xi$ is the vector of $P$, then at $P$

$$
\sum_{i} f_{i} d^{2} \xi_{i}=\kappa^{2} \mathscr{F} ; \quad \sum_{i} g_{i} d^{2} \xi_{i}=\kappa^{2} \mathscr{G} ; \quad \sum_{i} h_{i} d^{2} \xi_{i}=\kappa^{2} \mathscr{H} ;
$$

where $\kappa$ is a constant times $d t$.

2.6. We may evaluate $\sum_{i} f_{i} d^{3} \xi_{i}$ by the same method. A Laplace expansion gives from (13), (14) and the argument of Section 2.5

$$
\begin{aligned}
& \sum_{i, j} f_{i j} d^{2} \xi_{i} d \xi_{j}=-\kappa d \kappa \mathscr{F}+\kappa^{2}(A+B+D),
\end{aligned}
$$

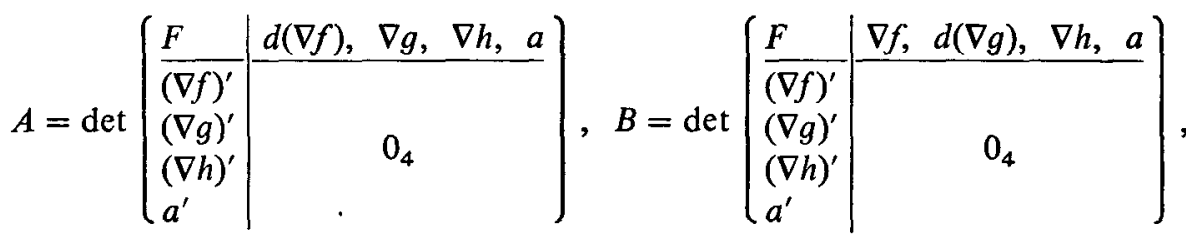

$$
\begin{aligned}
& D=\operatorname{det}\left(\begin{array}{l|c|}
F & \nabla f, \nabla g, d(\nabla h), a \\
\hline(\nabla f)^{\prime} & \\
(\nabla g)^{\prime} & 0 \\
(\nabla h)^{\prime} & \\
a^{\prime} &
\end{array}\right)
\end{aligned}
$$

By (5) and (12) the 6th column of $A$ is a linear combination of the preceding ones, so $A=0$. If we repeat the procedure of Section 2.5 on $B$ and $D$, and use (11) in addition to (1), (8) and (9) we obtain

$$
\begin{aligned}
& \sum_{i, j} f_{i j} d^{2} \xi_{i} d \xi_{j}=-\kappa d \kappa \mathscr{F}-\frac{\kappa^{2}}{(p-1)^{2}}\left\{\operatorname{det}\left(\begin{array}{l|l}
\frac{F}{(\nabla g)^{\prime}} \\
(\nabla h)^{\prime}
\end{array} \mid \frac{d(\nabla g), \quad \nabla h}{0_{2}}\right)\right. \\
& \left.+\operatorname{det}\left(\begin{array}{c|cc}
\frac{F}{(\nabla g)^{\prime}} & \frac{\nabla g,}{d(\nabla h)} \\
(\nabla h)^{\prime} & 0_{2}
\end{array}\right)\right\} .
\end{aligned}
$$

2.7. In order to deal with the second term in (18) we introduce some more notation. We write

$$
\begin{aligned}
& \overline{\mathscr{F}}_{i}=\frac{2}{(p-1)^{2}}\left\{\operatorname{det}\left(\begin{array}{l|l|l}
\frac{F}{(\nabla g)^{\prime}} \\
(\nabla h)^{\prime}
\end{array} \mid \begin{array}{cc}
\nabla g_{i} & \nabla h \\
0_{2}
\end{array}\right)+\operatorname{det}\left(\begin{array}{c|c}
\frac{\nabla g}{(\nabla g)^{\prime}} & \nabla h_{i} \\
(\nabla h)^{\prime} & 0_{2}
\end{array}\right)\right\} . \\
& \bar{\nabla} \mathscr{F}=\left(\overline{\mathscr{F}}_{0}, \overline{\mathscr{F}}_{1}, \overline{\mathscr{F}}_{2}, \overline{\mathscr{F}}_{3}, \overline{\mathscr{F}}_{4}\right)^{\prime} .
\end{aligned}
$$

If $f(x)$ is a quadratic then $\overline{\mathscr{F}}_{i}=\mathscr{F}_{i}$ and $\bar{\nabla} \mathscr{F}=\nabla \mathscr{F}$ : this is seen by taking a Laplace expansion of $\mathscr{F}$ by the last two rows and columns. This explains our choice of notation. $\bar{\nabla} \mathscr{G}$ and $\nabla \mathscr{H}$ are obtained similarly. 
2.8. Writing (18) in the form

$$
\Sigma f_{i j} d^{2} \xi_{i} d \xi_{j}=-\kappa d \kappa \mathscr{F}-\frac{\kappa^{2}}{2}(\bar{\nabla} \mathscr{F})^{\prime} d \xi
$$

it follows, by (6), (7), (16), that

$$
\sum_{i} f_{i} d^{3} \xi_{i}=3 \kappa d \kappa \mathscr{F}+\kappa^{2}\left(\nabla \mathscr{F}+\frac{1}{2} \bar{\nabla} \mathscr{F}\right)^{\prime} d \xi
$$

The analogues of (16) and (21) for $g$ and $h$, and an appeal to (13), show that $\left[(\nabla g)^{\prime} d^{2} \xi\right]\left[(\nabla h)^{\prime} d^{3} \xi\right]-\left[(\nabla h)^{\prime} d^{2} \xi\right]\left[(\nabla g)^{\prime} d^{3} d \xi\right]=$

$$
\kappa^{5} \operatorname{det}\left(\begin{array}{cccccc}
\mathscr{G}, & \mathscr{H}, & 0, & 0, & 0, & 0 \\
\nabla \mathscr{\nabla}+\frac{1}{2} \tilde{\nabla} \mathscr{G}, & \nabla \mathscr{H}+\frac{1}{2} \bar{\nabla} \mathscr{H}, & \nabla f, & \nabla g, & \nabla h, & a
\end{array}\right) .
$$

But from the analogues of (19) and (20) for $\mathscr{G}$ we have $\xi^{\prime} \bar{\nabla} \mathscr{G}=2(p+r-2) \mathscr{G}$, so from (10) we have $\xi^{\prime}\left(\nabla \mathscr{G}+\frac{1}{2} \bar{\nabla} \mathscr{G}\right)=3(p+q+r-4) \mathscr{G}$. The same relationship holds for $\mathscr{H}$. Subtract from the first row of the determinant in (22)

$$
(3 p+3 q+3 r-12)^{-1} \xi_{i}
$$

times the (i+1)th row for $i=0,1,2,3,4$. Using (1), (8) and putting $t=0$ we find

Lemma 2. If $\xi$ is the vector of $P$, then at $P$

$$
\begin{aligned}
{\left[(\nabla g)^{\prime} d^{2} \xi\right]\left[(\nabla h)^{\prime} d^{3} \xi\right]-\left[(\nabla h)^{\prime} d^{2} \xi\right]\left[(\nabla g)^{\prime} d^{3} \xi\right] } \\
=(3 p+3 q+3 r-12)^{-1} \kappa^{5} \operatorname{det}\left(\nabla \mathscr{G}+\frac{1}{2} \bar{\nabla} \mathscr{G}, \nabla \mathscr{H}+\frac{1}{2} \bar{\nabla} \mathscr{H}, \nabla f, \nabla g, \nabla h\right) .
\end{aligned}
$$

2.9. Lemmas 1,2 are the only results we shall need subsequently. They are true when the ground field $K$ is an arbitrary algebraically closed field of characteristic zero. We indicate the modifications necessary for their proof in this setting.

Instead of a branch of $C$ through $P$ we consider the single linear place with centre $P(17$, p. $193 ; 13$, p. 140$)$. We take a minimal parametrisation $\xi$ of this place satisfying (1), but now the $\xi_{i}$ are formal power series in an indeterminate $t$. We introduce a second indeterminate $d t$ to obtain differentials. Our formal calculations proceed as before, but we must modify the first paragraph of Section 2.4 which depends on convergence. $\xi$ is a generic point of $C(11, \mathrm{pp} .61$, $187)$, and so is simple. Thus $(\nabla f, \nabla g, \nabla h, a)$ has rank 4. By our hypothesis for $P$ there is an $i$ such that the "constant term" of the power series $|\nabla f, \nabla g, \nabla h, a|_{i}$ is non-zero. The inverse of this series is thus another power series. Equation (13) now follows, and hence so do all the numbered equations. Each of these equations is one in which all the terms are power series in $t$ times a power of $d t$. By taking the terms of zero order in $t$ in (16) and (22) we obtain Lemmas 1,2, where $\xi$ now stands for the centre of the place, $P$.

2.10. The osculating spaces to $C$ at $P$ are those of the branch or place. Suppose that the osculating plane at $P(\xi)$ has 3-point contact: the curve is not 
planar by Section 2.1. Then the osculating solid at $P$ is spanned by $\xi, d \xi, d^{2} \xi$, $d^{3} \xi$. This follows from (3, p. 199) however we regard the series (see also (11), p. 182). Any solid through $\xi$ and $d \xi$ has the form

$$
(\lambda \nabla f+\mu \nabla g+v \nabla h)^{\prime} x=0 .
$$

We obtain two independent equations for $(\lambda, \mu, v)$ when we require this solid to contain $d^{2} \xi$ and $d^{3} \xi$. By Lemma 1 the first of these is

$$
\kappa^{2}(\lambda \mathscr{F}+\mu \mathscr{G}+\nu \mathscr{H})=0 .
$$

Hence $\kappa$ is non-zero. From (23) and Lemma 2 we deduce

Theorem 1. Suppose that $C$ is an irreducible curve which is the complete intersection of the primals $f(x)=0 ; g(x)=0 ; h(x)=0 ;$ in an $S_{4}$ over an algebraically closed field of characteristic zero. Suppose that the threefolds have respective orders $p, q, r \geqq 2$, so that $C$ has order pqr. Let $P$ be a simple point on $C$ with coordinate $\xi$, and suppose that the osculating plane at $P$ has 3-point contact. THEN, with the definitions (4) and (20) above, the osculating solid at $P$ has equation

$$
\begin{aligned}
\left\{\nabla f \cdot \operatorname { d e t } \left(\nabla \mathscr{G}+\frac{1}{2} \bar{\nabla} \mathscr{G}, \nabla \mathscr{H}\right.\right. & \left.+\frac{1}{2} \bar{\nabla} \mathscr{H}, \nabla f, \nabla g, \nabla h\right) \\
& +\nabla g \cdot \operatorname{det}\left(\nabla \mathscr{H}+\frac{1}{2} \bar{\nabla} \mathscr{H}, \nabla \mathscr{F}+\frac{1}{2} \bar{\nabla} \mathscr{F}, \nabla f, \nabla g, \nabla h\right) \\
& \left.+\nabla h \cdot \operatorname{det}\left(\nabla \mathscr{F}+\frac{1}{2} \bar{\nabla} \mathscr{F}, \nabla \mathscr{G}+\frac{1}{2} \bar{\nabla} \mathscr{G}, \nabla f, \nabla g, \nabla h\right)\right\}^{\prime} x=0 .
\end{aligned}
$$

It is, perhaps, pertinent to point out that the result is true if $p=1$ but $q, r \geqq 2$, provided we put $\mathscr{F} \equiv 0$. The osculating solid is just the prime $f(x)=0^{\prime}$.

Our equation is of degree $6(p+q+r)-26$ in the $\xi_{i}$. Edge suggests (6, p. 279) that the degree should be $10(p+q+r-5)$, but he gives no indication of why this should be so. Our result implies that pqr $(6 p+6 q+6 r-26)$ osculating solids to $C$ pass through a general point $x$ : to see this interpret the equation of the osculating solid as a restriction on $\xi$ with $x$ fixed. We indicate an alternative derivation of this result for a general nonsingular $C$. Edge's factor $(p+q+r-5)$ appears in a formula for the genus. By $(12$, p. 191) the rank of $C$ is

$$
p q r(p+q+r-3)
$$

so its genus $D$ is $(12$, p. 190) given by

$$
2(D-1)=p q r(p+q+r-3)-2 p q r=p q r(p+q+r-5) \text {. }
$$

The number of osculating solids to $C$ through $x$ equals the number of sets of a linear series $g_{p q r}^{3}$ that have four coincident members, where the series is cut on $C$ by the solids through $x$. This number is $(4$, p. $10 ; 12$, p. 389)

$$
4\{p q r+3(D-1)\} \text {, }
$$

which by (25) is $p q r\{4+6(p+q+r-5)\}$ or $p q r(6 p+6 q+6 r-26)$. Thus the equation of the osculating solid at $\xi$ has degree $6(p+q+r)-26$ in the $\xi_{i}$ this corrects the $10(p+q+r-5)$ of (6). 


\section{The intersection of three quadrics in $S_{4}$}

3.1. Suppose that each threefold is a quadric and that

$$
f(x)=x^{\prime} A x, \quad g(x)=x^{\prime} B x, \quad h(x)=x^{\prime} C x .
$$

$C$ is then an octavic of genus 5 , the canonical model for curves of genus 5 . Each point $\xi$ of $C$ is nonsingular or simple (11, p. 279; 13, p. 188). From (4) we find that

$$
\mathscr{F} \equiv 2^{7} \operatorname{det}\left(\begin{array}{c|cc}
\boldsymbol{A} & \boldsymbol{B} \xi, \quad \boldsymbol{C} \xi \\
\bar{\xi}^{\prime} \boldsymbol{B} & \mathbf{0}_{2}
\end{array}\right)=2^{7} \mathscr{A} \text { say. }
$$

$\mathscr{B}$ and $\mathscr{C}$ are similarly defined as $2^{-7} \mathscr{G}$ and $2^{-7} \mathscr{H}$ respectively. Recalling from Section 2.7 that $\bar{\nabla} \mathscr{F}$ and $\nabla \mathscr{F}$ now coincide we obtain

Theorem 2. Suppose that the point $\xi$ of $S_{4}$ satisfies

$$
f \equiv \boldsymbol{\xi}^{\prime} \boldsymbol{A} \boldsymbol{\xi}=0 ; \quad g \equiv \boldsymbol{\xi}^{\prime} \boldsymbol{B} \xi=0 ; \quad h \equiv \boldsymbol{\zeta}^{\prime} \boldsymbol{C} \xi=0
$$

where the quadrics intersect in a canonical curve $C$ of genus 5. Define

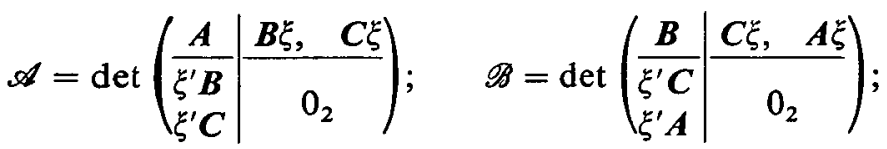

$$
\begin{aligned}
& \mathscr{C}=\operatorname{det}\left(\begin{array}{c|cc}
\boldsymbol{C} & \boldsymbol{A} \xi, \quad \boldsymbol{B} \xi \\
\hline \bar{\zeta}^{\prime} \boldsymbol{A} & 0_{2}
\end{array}\right) .
\end{aligned}
$$

Then, if the osculating plane to $C$ at $\xi$ has 3-point contact, the equation of the osculating solid to $C$ at $\xi$ is

$$
\begin{aligned}
\xi^{\prime}\left\{A \cdot \frac{\partial(\mathscr{B}, \mathscr{C}, f, g, h)}{\partial\left(\breve{\zeta}_{0}, \xi_{1}, \xi_{2}, \xi_{3}, \xi_{4}\right)}+\boldsymbol{B} \cdot \frac{\partial(\mathscr{C}, \mathscr{A}, f, g, h)}{\partial\left(\xi_{0}, \xi_{1}, \xi_{2}, \xi_{3}, \xi_{4}\right)}\right. & \\
& \left.+C \cdot \frac{\partial(\mathscr{A}, \mathscr{B}, f, g, h)}{\partial\left(\xi_{0}, \xi_{1}, \xi_{2}, \xi_{3}, \xi_{4}\right)}\right\} x=0 .
\end{aligned}
$$

3.2. We make a few comments about the relationship of the quadrics to the quartic primal

$$
\operatorname{det}\left(\begin{array}{c|cc}
A & B x, \quad C x \\
\hline x^{\prime} \boldsymbol{B} & 0_{2} \\
x^{\prime} \boldsymbol{C} &
\end{array}\right)=0 .
$$

A point $x$ lies on the quartic if and only if there are $y, \mu, v$ not all zero such that

$$
A y+\mu B x+\nu C x=0 ; \quad x^{\prime} B y=x^{\prime} C y=0 .
$$

Certainly each of the 5 vertices of the common self-polar simplex of the quadrics $g$ and $h$ lies on the quartic-we restrict our attention to the general case. For 
at each vertex $x$ the vectors $B x, C x$ are dependent. Moreover, each such $x$ is a singular point on the quartic: each term in the sum obtained on differentiating the left-hand side of (28) has either its last two rows or its last two columns dependent.

Suppose $x$ is on the quartic distinct from these points. Then from (29) $y \neq 0$ and gives a point in the polar plane $\pi$ of $x$ with respect to the pencil of quadrics determined by $g$ and $h$. Further, the polar solid of $y$ with respect to $f$ contains $\pi$. From (29) we obtain

$$
y^{\prime} A y=0,
$$

so $y$ is on $f$. Hence $\pi$ meets $f$ in a singular conic, and $y$ is a vertex. Conversely, suppose that $x$ is a point whose polar plane $\pi$ with respect to the pencil $g, h$ has a singular section with $f$. Let $y$ be a vertex of the section. Then $y$ is conjugate to each point of $\pi$ with respect to $f$, and (29) follows. Hence $x$ lies on the quartic.

Suppose, now, that $x$ is a common point of $C$ and the quartic. The points $x$ and $y$ do not coincide: if they did then by (29) the tangent solids to the quadrics at $x$ would meet in a plane, and $x$ would not be simple on $C$. Since $x$ is in $\pi$ it is conjugate to $y$ with respect to $f$. Hence $x y$ is the tangent line to $C$ at $x$ and lies on $f$. Conversely, suppose that the tangent line to $C$ at a point $x$ lies on $f$. This line lies in $\pi$, which thus has a singular section with $f$. Thus $x$ is on the quartic. We conclude that there are $4 \times 8=32$ points of $C$ whose tangent lines are lines on $f$. Our quartic determines these points. The number of tangents to $C$ which lie on $f$ can be obtained also from a formula given by Baker (1, p. 23). He uses the Cayley-Brill formula to deduce that a nonsingular curve with order $n$ and genus $D$ which lies on a quadric has $2(n+2 D-2)$ of its tangents on the quadric. Taking $n=8$ and $D=5$ for $C$ gives 32. I am indebted to the referee for this reference, and for other helpful remarks.

3.3. To obtain Edge's curve we take $A=I_{5}, B=$ diag. $\left(a_{0}, a_{1}, a_{2}, a_{3}, a_{4}\right)$ and $C=B^{2}$, where the $a_{i}$ are distinct and non-zero (6, p. 277). Suppose that the osculating plane at $\xi$ on $C$ has 3-point contact. After some tedious manipulation we find from Theorem 2 that the osculating solid at $\xi$ has equation where $\sigma=\sum_{j} a_{j}$.

$$
\xi_{0} \xi_{1} \xi_{2} \xi_{3} \xi_{4}\left[\sum_{i}\left\{\sum_{j} a_{j}^{4} \xi_{j}^{2}-\left(\sigma-a_{i}\right) \sum_{j} a_{j}^{3} \xi_{j}^{2}\right\}^{2} \xi_{i} x_{i}\right]=0
$$

One way of carrying out the calculation is as follows. Each of $\mathscr{A}, \mathscr{B}, \mathscr{C}$, consists of a diagonal matrix bordered by monomials. Taking a double Laplace expansion by its last two rows and columns shows that $\mathscr{Z}$ is a linear combination of the products $\xi_{i}^{2} \xi_{j}^{2}$ with $i \neq j$. The same is true for $\mathscr{B}$ and $\mathscr{C}$. Thus all three Jacobians in Theorem 2 have $\xi_{l}$ for a factor along the $(i+1)$ th (or $\partial / \partial \xi_{i}$ th) row. This shows that each Jacobian has a factor $\xi_{0} \xi_{1} \xi_{2} \xi_{3} \xi_{4}$. The residues have three rows of constants and two of linear combinations of the $\xi_{j}^{2}$. Hence what is in \{\}$^{2}$ must be quadratic in these squares. When one 
performs this procedure in detail the outcome is (31), once an insignificant numerical factor is removed.

We deduce from (31) that the osculating planes to $C$ at its intersections with primes of the simplex of reference have more than 3-point contact. Edge knew this in advance $(6$, p. $279 ; 7$, p. 486). For the other points of $C$ he was able to find the osculating solids without the unnecessary factor $\xi_{0} \xi_{1} \xi_{2} \xi_{3} \xi_{4}$.

\section{Some special curves of intersection in $S_{n}$}

4.1. We consider $n-1$ primals of order $m$ given by

$$
\sum_{i} a_{i}^{(j)} x_{i}^{m}=0, j=0,1, \ldots, n-2 .
$$

Summations now run over $0,1,2, \ldots, n$. We assume that each $(n-1)$-square submatrix of the $(n-1) \times(n+1)$ matrix with jith entry $a_{i}^{(j)}$ is nonsingular. Then any common point of the primals has at most one coordinate zero, and so at such a point the primals have independent tangent $S_{n-1}$. Their intersection $C$ is thus an irreducible nonsingular curve of order $m^{n-1}$. Regarding, for the moment, $\left(a^{(0)}, a^{(1)}, \ldots, a^{(n-2)}\right)$ as coordinates of an $S_{n-2}$ the condition implies that no $n-1$ of $\left(a_{i}^{(0)}, a_{i}^{(1)}, \ldots, a_{i}^{(n-2)}\right)$ are coprimal. Hence there is a unique rational normal $(n-2)$-ic through these $n+1$ points, which may be taken as $\left(1, t, t^{2}, \ldots, t^{n-2}\right)$ by an appropriate choice of the reference system (cf. (8), p. 261). Thus $C$ is determined by

$$
\sum_{i} a_{i}^{j} x_{i}^{m}=0, j=0,1, \ldots, n-2,
$$

where the $a_{i}$ are distinct, since we may take any $n-1$ independent members of the linear system determined by the primals of (32) as a base for $C$. When $m=2$ the curve $C$ is the intersection of quadrics with a common self-polar simplex. This curve occurs in (2, p. 185): for $n=4$ it is Edge's curve, for $n=5$ it is the image by the Klein representation of the tangents to an inflexional curve of the Kummer surface (2, pp. 230, 231).

Denote by $X_{i}$ that vertex of the simplex of reference whose $i$ th coordinate is 1 , and let $\pi_{i}$ be the opposite face. $C$ meets $\pi_{i}$ in $m^{n-1}$ points. If $P$ is a point on $C$ not in $\pi_{i}$ then the line $X_{i} P$ has $m-1$ further intersections with $C$ : they are obtained by replacing the $i$ th coordinate in a vector for $P$ by $\rho$ times this coordinate where $\rho \neq 1$ is an $m$ th root of unity. If, on the other hand, $W$ is in both $C$ and $\pi_{i}$ these $(m-1)$ further intersections on $W X_{i}$ coincide with $W$ : the tangent line to $C$ at $W$ is $W X_{i}$ having contact of order $m$. The osculating plane at $W$ is the limiting position of the plane $X_{i} W P$ as $P$ approaches $W$ along $C$. This plane meets $C$ in $m$ intersections at $W$ and $m$ intersections on $X_{i} P$. Each of the intersections has $W$ as its limiting position, so the osculating plane has $2 m$-point contact. Continuing in the same fashion-the osculating solid is the limit of the join of the osculating plane to $P$-we find that the osculating $S_{r}$ at $W$ has mr-point contact. This parallels (7, pp. 486, 487). In the notation of 
(3, p. 199) the indices $l_{1}, l_{2}, \ldots, l_{n}$ of the branch of $C$ through $W$ are $0, m-2$, $2(m-1)-1,3(m-1)-1, \ldots,(n-1)(m-1)-1$ respectively.

The primes of $S_{n}$ through a general point $Q$ cut on $C$ a linear series $g_{m^{n-1}}^{n-1}$. The same calculation as in Section 2.10 shows that the number of sets of this series that have $n$ coincident members is

$$
\frac{1}{2} n m^{n-1}\left[(n-1)^{2}(m-1)-2 n+4\right] \text {. }
$$

The genus $D$ of $C$ is given by

$$
2(D-1)=m^{n-1}[(n-1)(m-1)-2] .
$$

The join of $Q$ to the osculating $S_{n-2}$ at a $W$ counts as

$$
l_{1}+l_{2}+\ldots+l_{n-1}=\frac{(n-2)}{2}[(n-1)(m-1)-2]
$$

towards this number (3, p. 200). Hence there are through $Q m^{n-1}[(n-1) m-1]$ primes with $n$-point contact (at least) with $C$ and not so related to the $W$. We shall show below that there are precisely this number of osculating primes through $P$ whose corresponding osculating $S_{n-2}$ have $(n-1)$-point contact. Further, each $W$ contributes

$$
l_{1}+l_{2}+\ldots+l_{n}=\frac{(n-1)}{2}[n(m-1)-2]
$$

towards the number of hyperosculating $S_{n-1}$ to $C$. Thus the $W$ together contribute all the $(3$, p. $200 ; 12$, p. 388$)(n+1)\left[m^{n-1}+n(D-1)\right]$ hyperosculating $S_{n-1}$ of $C$. The $W$ are thus the only exceptional points of $C$ : the situation echoes that for Edge's curve.

4.2. Let $P$ be a point on $C$ whose osculating $S_{n-2}$ has $(n-1)$-point contact. We find the osculating $S_{n-1}$ at $P$ be a generalisation and slight modification of Edge's method.

We define

$$
f(\theta)=\left(\theta-a_{0}\right)\left(\theta-a_{1}\right) \ldots\left(\theta-a_{n}\right) ; \quad s_{r}=\sum_{r} \frac{a_{i}^{r}}{f^{\prime}\left(a_{i}\right)},
$$

where dashes now denote derivatives. We then have

$$
s_{r}=0, r=0,1, \ldots, n-1 ; \quad s_{n}=1 ; \quad s_{n+1}=\sum_{i} a_{i}=\sigma, \text { say } .
$$

To see this consider the partial fractions for $\theta^{r+1} / f(\theta)$. Regarding (33) as independent linear equations for the $x_{i}^{m}$ we see from (38) that the points $\xi$ on $C$ are given by

$$
f^{\prime}\left(a_{i}\right) \xi_{i}^{m}=p+a_{i} q, \quad i=0,1, \ldots, n
$$

for arbitrary $p, q$. A differentiation gives

$$
m f^{\prime}\left(a_{i}\right) \xi_{i}^{m-1} d \xi_{i}=d p+a_{i} d q .
$$


Using (40) and a straightforward induction argument we easily obtain, for the points of $C$ not in any of the $\pi_{i}$,

$$
f^{\prime}\left(a_{i}\right) \xi_{i}^{m-1} d^{r} \xi_{i}=C_{r} \frac{F_{r}\left(a_{i} ; p, q\right)}{\left(p+a_{i} q\right)^{r-1}}, \quad r \geqq 1, \quad i=0,1, \ldots, n ;
$$

where $C_{r}$ is a constant, and $F_{r}(\theta ; p, q)$ is a polynomial in $\theta$ of degree $\leqq r$ whose coefficients are sums of products of powers of differentials of $p$ and $q$.

Take, now, $\xi$ to be the coordinate vector of $P$. The osculating $S_{n-1}$ at $P$ is spanned by $\xi, d \xi, d^{2} \xi, \ldots, d^{n-1} \xi$. But by (38), (39) and (4l) we see that these all lie in the prime

$$
\sum_{i}\left(p+a_{i} q\right)^{n-2} \xi_{i}^{m-1} x_{i}=0 .
$$

From (38) and (39) we have

$$
\sum_{i} a_{j}^{n-1} \xi_{j}^{m}=q ; \quad \sum_{j} a_{j}^{n} \xi_{j}^{m}=p+\sigma q ;
$$

so the osculating $S_{n-1}$ is

$$
\sum_{i}\left\{\sum_{j} a_{j}^{n} \xi_{j}^{m}-\left(\sigma-a_{i}\right) \sum_{j} a_{j}^{n-1} \xi_{j}^{m}\right\}^{n-2} \xi_{i}^{m-1} x_{i}=0 .
$$

This has degree $m(n-1)-1$ in the $\xi_{i}$, so this times $m^{n-1}$ is the number of such osculating $S_{n-1}$ which pass through a general point $x$; as we claimed in Section 1 .

In a recent letter Edge informs me that he has verified a variant of this equation for the quadric case $m=2$.

\section{Concluding remarks}

We may consider in $S_{n}$ an irreducible curve which is the complete intersection of $(n-1)$ general primals $f^{(l)}(x)=0$ for $l=1,2, \ldots,(n-1)$. The methods of Section 2 together with a little extra determinantal theory give in succession the osculating plane and solid at a point $\xi$ of the curve. We may take the obvious extension of the notation of Section 2. If the tangent line at $\xi$ has 2-point contact then we find that the osculating plane there has its dual plane coordinates $\pi_{i j \ldots}$ given by

$$
\pi_{i j \ldots}=\operatorname{det}\left(\begin{array}{cccc}
\mathscr{F}^{(1)} & f_{i}^{(1)} & f_{j}^{(1)} & \ldots \\
\mathscr{F}^{(2)} & f_{i}^{(2)} & f_{j}^{(2)} & \ldots \\
\vdots & \vdots & \vdots & \ldots \\
\mathscr{F}^{(n-1)} & f_{i}^{(n-1)} & f_{j}^{(n-1)} & \ldots
\end{array}\right) .
$$

If this plane has 3-point contact at $\xi$ then we find that the dual solid coordinates $\omega_{i j \ldots}$ of the osculating solid at $\xi$ are given by

$$
\begin{aligned}
\omega_{i j \ldots}= & \sum_{1 \leqq k<l \leqq n-1}(-1)^{k+l+1}\left\{L _ { k l } \operatorname { d e t } \left(\bar{\nabla} \mathscr{F}^{(k)}+\frac{1}{2} \bar{\nabla} \mathscr{F}^{(k)},\right.\right. \\
& \left.\left.\quad \bar{\nabla} \mathscr{F}^{(l)}+\frac{1}{2} \nabla \mathscr{F}^{(l)}, \nabla f^{(1)}, \ldots, \bar{\nabla} f^{(n-1)}\right)\right\},
\end{aligned}
$$


where $L_{k l}$ is the determinant of the matrix obtained by removing $k$ th and $l$ th rows of

$$
\left(\begin{array}{ccc}
f_{i}^{(1)} & f_{j}^{(1)} & \ldots \\
f_{i}^{(2)} & f_{j}^{(2)} & \ldots \\
\vdots & \vdots & \ldots \\
f_{i}^{(n-1)} & f_{j}^{(n-1)} & \ldots
\end{array}\right)
$$

These are the natural generalisations to $S_{n}$ of Hesse's and our results as far as algebraic method is concerned. But there is another natural, and more important, geometric extension: we should like to have the equation of the osculating $S_{n-1}$ at a point on the general curve of intersection of $(n-1)$ primals in $S_{n}$. This problem presents formidable algebraic difficulties even when $n=5$. Fourth-order differentials are involved, and it is they that cause trouble.

The same difficulties occur in $S_{4}$ when attempting to find a primal cutting our curve $C$ in its points of hyperosculation. These complications remain in the particular case of the octavic intersection of three quadrics. The points of hyperosculation are then the 120 Weierstrass points. There ought to be a combinantal primal of the net of quadrics through $C$ which cuts $C$ in these points. It remains to find such a primal of degree 15 .

\section{REFERENCES}

(1) H. F. BAKER, On general curves lying on a quadric, Proc. Edinburgh Math. Soc. (2) 1 (1927), 19-30.

(2) H. F. BAKER, Principles of Geometry, Vol. 4 (Cambridge, 1925).

(3) H. F. BAKER, Principles of Geometry, Vol. 5 (Cambridge, 1933).

(4) H. F. BAKER, Principles of Geometry, Vol. 6 (Cambridge, 1933).

(5) A. Clebsch, Ueber die Wendungsberührebenen der Raumcurven, J. Reine Angew. Math. 63 (1864), 1-8.

(6) W. L. EdGe, The osculating solid of a certain curve in [4], Proc. Edinburgh Math. Soc. (II) 17 (1971), 277-280.

(7) W. L. Edge, Humbert's plane sextics of genus 5, Proc. Cambridge Philos. Soc. 47 (1951), 483-495.

(8) W. L. Edge, The tacnodal form of Humbert's sextic, Proc. Royal Soc. Edinburgh Sect. A 68 (1970), 257-269.

(9) O. Hesse, Über die Wendepunkte der algebraischen ebenen Kurven und die Schmeigungs-Ebenen der Kurven von doppelter Krummung, welche durch den Schnitt zweier algebraischen Oberfiächen entstehen, J. Reine Angew. Math. 41 (1 851), 272-284. 
(10) G. Salmon, A Treatise on the Analytic Geometry of Three Dimensions (Dublin, 1882).

(11) J. G. Semple and G. T. Kneebone, Algebraic Curves (Oxford, 1959).

(12) J. G. SEMPLE and L. Roth, Introduction to Algebraic Geometry (Oxford, 1949).

(13) R. J. WALker, Algebraic Curves (Princeton, 1950).

School of Mathematics

UNIVERSITY OF NEWCASTLE UPON TYNE 